
\title{
25 Research Square \\ Is YouTube a Reliable Source of Health-Related Information? A Systematic Review
}

\author{
Khalifa University \\ Fatma Mohamed \\ Khalifa University \\ Mohamed Elhassan \\ Saint Agnes Medical Center \\ Abdulhadi Shoufan \\ Khalifa University
}

Wael Osman ( $\nabla$ wael.osman@ku.ac.ae)

\section{Research Article}

Keywords: YouTube, Health information, Medical videos, Quality assessment, Bias

Posted Date: November 15th, 2021

DOI: https://doi.org/10.21203/rs.3.rs-1047141/v1

License: (9) This work is licensed under a Creative Commons Attribution 4.0 International License. Read Full License 


\section{Abstract}

Background: You Tube is a valuable source of health-related educational material which can have a profound impact on people's health-related behaviors and decisions. However, YouTube contains a wide variety of unverified content that may promote unhealthy behaviors and activities. We aim in this systematic review to provide insight into the published literature concerning the quality of health information and educational videos found on YouTube.

Methods: A search of peer-reviewed original articles was conducted regarding the educational value of YouTube medical videos which were published in English. We searched Google Scholar, Medline (through PubMed), EMBASE, Scopus, Direct Science, Web of Science, and ProQuest databases. A literature search was conducted between April 1 and April 31 of 2021. Based on the eligibility criteria, 202 artilces covering 30 medical categories were included in the qualitative synthesis.

Results: We reviewed approximately 22,300 videos in all of the studies we reviewed, with a mean of 115 videos per paper, which were assessed by 2.6 assessors. The quality of the videos was rated based on either scores or categories. Researchers commonly employed scoring systems that were either standardized (e.g., GQS, DISCERN, and JAMA) or based upon the guidelines and recommendations of professional associations. The results using different scores indicated an average to below-average quality. According to our aggregate quality assessment data, most of the videos were of poor quality. In terms of bias, only $32 \%$ of the videos appear neutral toward the health content, indicating that bias occurs frequently in these videos. Furthermore, the majority of the studies found a negative correlation between the quality and popularity of the videos.

Conclusions: YouTube is currently a poor to average source of health information. Furthermore, YouTube metrics are indeed tricky and can be misleading when determining the quality of videos. The future solution should be to implement ranking filtering algorithms that consider experts' endorsements, peerreview, and other metrics that can push up endorsed videos in the search results.

\section{Background}

YouTube is currently the second most popular search engine and social media platform. Youtube, which was founded in 2005, had over 2.1 billion users in 2020, resulting in over one billion hours of video being viewed each day and over 500 hours of video being uploaded each minute ${ }^{1}$. According to estimates, over $95 \%$ of the Internet population from 88 countries and 76 languages engages in some form of social interaction on YouTube every week (e.g., liking, sharing, and commenting) ${ }^{2,3}$. The United States alone is estimated to have $74 \%$ of the adult population using YouTube by September 20204 .

The instant popularity of YouTube is due to multiple factors, including its accessibility to almost any type of content in a simple and highly efficient mobile application, its popularity among young people 3,5 including young trainees and residents ${ }^{6}$, as well as its sharing of its enormous advertising revenue with 
the content creators (also known as YouTubers). Moreover, since $70 \%$ of users access YouTube using their mobile devices ${ }^{7}$, YouTube can be accessed at any time of the day.

Nowadays, YouTube has emerged as a valuable educational resource as a result of its innovative digitalization. Specifically, the YouTube model is based on a visual model that can include both theory and practice for teaching purposes. YouTube's popularity, ease of access, and relatively uncontrolled nature also make it a powerful tool for influencing people's decisions and promoting their well-being. For example, Mamlin and colleagues (2016) predicted that social media platforms such as YouTube would be widely used to exchange healthcare information between healthcare providers and consumers, to provide peer-to-peer patient support, and as a tool for public health surveillance ${ }^{8}$. The health information videos on YouTube are derived from various sources, such as those uploaded by doctors and health institutions, universities and medical schools, patients, advertisers, and user-generated content. However, and regardeless of the source of the content, YouTube's terms of service stipulate that "the content is the responsibility of the person or entity that provides it to the Service" ${ }^{9}$. YouTube's search results are typically based on metrics such as views, likes, and dislikes, rather than quality-based metrics, which leads to the wide availability of misleading videos that promote unhealthy habits and activities or provide inaccurate information ${ }^{10}$. For example, a recent study found that more than $25 \%$ of the most viewed YouTube videos regarding COVID-19 contained misleading information that reached millions of people around the world ${ }^{11}$. Furthermore, Nour and colleagues (2016) reported that inaccurate and accurate YouTube videos both received similar views for receiving information about a particular subject such as psoriasis $^{12}$.

A limited number of reviews have evaluated the quality of health information available on YouTube, either in general ${ }^{13,14}$ or concentrating on specific topics like surgical education ${ }^{15}$. According to these reviews, there was a lack of standardization and unclear information regarding patient education on YouTube. There were a limited number of articles included in the reviews, ranging from seven to 18 , which did not provide an elaborate description of the problem or provide detailed recommendations.

The current study examined 202 articles from 30 general health fields providing health information on YouTube. We provide detailed information regarding the scores used to evaluate each article's YouTube video content and the bias sources. Furthermore, we provide recommendations from the articles which we believe will help provide practical solutions to improve YouTube's educational value for health information.

\section{Methods}

\subsection{Literature search:}

A flowchart of the search strategy and papers selection can be seen in Fig. 1. The search was conducted using Google Scholar, Medline (through PubMed), EMBASE, Scopus, Direct Science, and Web of Science databases from April 1st through April 31st, 2021. We also searched the ProQuest database for 
dissertations and theses in order to avoid publication bias. As we observed a noticeable shift towards papers on the COVID-19 pandemic after that, which may affect our conclusions, we limited the search to papers published by August 2020 to ensure unbiased coverage of health information topics. The search terms used included "YouTube" and "medical, medical education, health, healthcare, health information," and "quality." Two researchers (FA, WO) conducted the search independently. Any disagreements were resolved by a third reviewer (AS).

\subsection{Eligibility and papers selection:}

First, we read the abstracts or scanned the full texts and supplementary materials if the abstracts were not sufficiently informative. Based on this, we classified the eligibility as yes, no, or unclear and included the unclear abstracts until we reviewed the full-text articles. The inclusion criteria for the papers were: peer-reviewed original articles about the quality of educational value of YouTube medical videos published by the end of August 2020 in English. The exclusion criteria included papers that did not meet the inclusion criteria, duplicate publications, technical reports, organization websites, case reports, and organizational reports. In reviewing the full-texts, we reviewed the five theses ${ }^{16-20}$; but we excluded three of them from this study since they presented descriptive analyses of health topics without assessing the quality of the research as an eligibility criterion. Ultimately, we selected 202 artilces to be reviewed, the titles and topics of which are summarized in the Supplementary Table 1.

\subsection{Data extraction:}

From the included papers, we collected the following data: title, abstract, topic, quality assessment score and results, number of assessors, number of videos, the resulting categories and classifications, type and source of bias, and conclusion and recommendations. Data extraction and analysis were conducted using the PRISMA recommendations for systematic reviews (Fig. 1).

\subsection{Statistical analysis:}

We used simple descriptive statistics like means, standard deviations, ranges, and frequency to describe the data.

\section{Results}

\subsection{Methodological aspects:}

In our review, we selected 202 papers to be reviewed (Fig. 1, Methods), the titles and topics of which are summarized in the Supplementary Table 1. We found that researchers adopted different approaches to evaluate the quality of health information on YouTube, but they generally followed these steps:

1. Focus on a single topic, such as a particular disease or treatment

2. A cross-sectional analysis of related videos and the application of some inclusion criteria, such as being in English or having a minimum number of views 
3. Videos are evaluated by one or more experts, usually among the authors themselves. Researchers in some studies evaluated the scoring reliability using an inter-rater agreement analysis, resolved discrepancies by consensus, or invited an additional assessor to settle disagreements.

4. Assessors used a scoring system either developed by the authors or standardized by the assessors

5. According to the scores, videos were assigned to different quality categories

6. According to the results of the scoring or classification process, authors generally evaluate the suitability of YouTube for providing reliable health-related information

7. Based on this judgment, some general remarks and recommendations were made.

We summarize the results of our review in the following sections in light of this general approach. We first highlight some methodological aspects and then summarize the findings of reviewed papers.

In Table 1, we summarize some statistics related to the number of videos, assessors, and quality categories included in the reviewed studies. Across all the studies we reviewed, the total number of videos was approximately 22,300, with an average of 115 videos per paper, assessed by an average of 2.6 assessors. The large number of quality categories makes it difficult to aggregate the data. As an example, some studies categorize the videos into three classes, for example "excellent", "moderate", and "poor" 21, whereas other studies use four categories, for example "very useful", "useful", "slightly useful", or "misleading" 22.

Table 1

The basic metrics for quality assessment of health-related content in studies

\begin{tabular}{|llllll|}
\hline Feature & Mean & SD & Median & Max & Min \\
\hline Number of videos & 115 & 116 & 96 & 1000 & 2 \\
\hline Number of assessors & 2.6 & 1.2 & 2 & 8 & 1 \\
\hline Number of quality classes & 3.2 & 0.9 & 3 & 6 & 2 \\
\hline
\end{tabular}

In general, each paper addressed a series of videos related to a single topic. In a few instances, the same subject was addressed in more than one study. Therefore, the reviewed papers cover a wide range of topics. To provide a concise overview of these topics, we classified them into 30 different medical categories and determined the number of studies under each category, as shown in Fig. 2.

The majority of authors frequently used their scoring systems based on some reference in the respective medical field in order to assess the quality of the content (Fig. 3). For instance, Brooks and colleagues (2014) based their evaluation of videos about patient information for lumbar discectomy on the recommendations of the British Association of Spine Surgeons ${ }^{23}$. Aside from these, general quality standards were used by many authors. These include the Global Quality Standard (GQS), the DISCERN 
instrument, and the Journal of the American Medical Association (JAMA) benchmark criteria. These three scores are described elsewhere 24,25 .

\subsection{Quality assessment results}

The reviewed studies assessed content quality by scoring, categorizing, or both. In addition, many authors correlated quality metrics with video popularity metrics such as views and likes. Nearly all reviewed papers conclude with recommendations. In the following, we present the findings of the reviewed studies according to these aspects.

The quality assessment by scores presents the average quality score of videos according to the three most widely used standards GQS, DISCERN, and JAMA (Fig. 4). As an example, 25 papers used the GQS standard and provided a mean score for each video. The value of 2.68 in Fig. 4 is the average of the 25 mean values presented in related papers. As can be seen in the figure, the mean score is the same for all three standards, noting the 4-point scoring system of JAMA as opposed to the 5-point scoring systems used for the other two standards.

The majority of papers assessing content quality by classification use category labels to describe quality, usefulness, or reliability of the quality assessment. In order to facilitate data aggregation, we have mapped the used category names to one of five labels, as shown in Table 2. The percentage of videos which are assigned to the categories "excellent," "very useful," "very accurate," or "high quality" in the reviewed studies is grouped under the category "excellent quality" in our study.

Table 2

Assigning quality categories in reviewed papers to five categories for the purposes of data aggregation

\begin{tabular}{|lllll|}
\hline $\begin{array}{l}\text { Excellent } \\
\text { quality }\end{array}$ & Good quality & Average quality & Not useful & Poor quality \\
\hline - Excellent & - Educationally & - Fair & - Not educationally & - Dangerously \\
- Very useful & useful & misleading \\
- Very & - Useful & - Satisfactory & - Irrelevant & - Misleading \\
accurate & - Good & - Slightly useful & - Offering little value & - Very poor \\
- High & - Accurate & - Somewhat & & - Poor \\
quality & - Reliable & - Moderately & & - Inadequate \\
& & useful & & - Low quality \\
& & - Intermediate & & - Not reliable \\
& & quality & - Inaccurate \\
\hline
\end{tabular}

Figure 5 illustrates the results of data aggregation. The upper dark bars represent the average percentage of videos classified into each category. For example, the value of $40 \%$ in the figure represents the average 
percentage of videos assigned to the category " poor" within the reviewed papers. Lower light bars indicate the relative frequency of using the categories in the related studies. As a result, " not useful "was the most frequently used category, followed closely by "poor quality" and "good quality," with "excellent quality" being the least frequently used.

In some papers, controversial topics were discussed, such as vaccination ${ }^{26}$ or unauthorized treatments 27. In such cases, the authors categorized the videos based on the producers' perceived bias towards or against the topic, as determined by the authors, and the results are summarized in Fig. 6 . According to the figure, the average proportion of videos is $58 \%$ in favor of the discussed treatment. A majority of the videos imply commercial interest (51\%), while only $32 \%$ are neutral by highlighting the advantages and disadvantages of presented topics without supporting or devaluing them.

Almost a third of the papers correlate the quality of analyzed videos with their popularity metrics, such as views, likes, dislikes, shares, and comments. Fig. 7 summarized the results of these analyses. For example, 3 of the studies found no correlation and 13 found a negative correlation between number of views and quality of the videos ${ }^{28,29}$, meaning that lower-quality videos were viewed more frequently than higher-quality videos ${ }^{30,31}$. In only seven papers, a positive correlation was observed between the quality and popularity of both views and likes ${ }^{32,33}$.

Furthermore, some papers classified videos according to their comprehensiveness, i.e., the amount of coverage that was considered essential for each topic ${ }^{34-36}$. As an example, Pant and colleagues (2012) assessed the credibility of YouTube content on acute myocardial infarction and discovered that only 6 percent of the reviewed videos addressed all relevant aspects according to the authors' criteria ${ }^{37}$. The average percentage of total videos in all reviewed articles is $13.2 \%$.

Finally, based on their research findings, almost all reviewed papers provide one or more recommendations to improve the quality of health-related content on YouTube, as summarized in Fig. 8. Accordingly, most frequent recommendations (in 65\% of the papers) highlight the role of reputable sources such as professional societies, health organizations, academic institutions, medical institutions in providing qualified content on YouTube ${ }^{38-41} .13 \%$ the reviewed papers explicitly or implicitly regard the situation irredeemable and entirely discouraged using YouTube as a source for health-related information 42-46. Only a very few authors took the opposite position and recommended YouTube without concerns 47,48 .

\section{Discussion}

A reliable and up-to-date source of health information is essential, as it can significantly influence the health of users and their ability to make informed decisions about their treatment and medication. YouTube was originally introduced as a platform for entertainment and not education. Since then, it has become an indispensable educational resource for both the general public and specialists alike. As an example, different studies suggest that surgical videos on YouTube are currently the preferred source of 
education among trainees as they are free, portable, reusable, and accessible via mobile phones ${ }^{6,49}$. Accordingly, researchers expressed early concerns about the quality of health-related content on YouTube and began investigating the issue as early as $2007^{50}$. Since then, the number of publications that evaluate the quality of health-related content has steadily increased.

As shown in Fig. 2, our study included papers that evaluated YouTube's health-related information in almost all specialties. It is important to keep in mind that within each of the topics in Fig. 2, the videos covered a wide range of pathological conditions and health information (e.g., the orthopedics category may contain information on inflammatory conditions, cancer, or surgical procedures). Some specialties were covered more than others potentially because of their frequency, the difficulty of understanding the disease's nature, or because researchers in those specialties are more engaged in research on the subject. In general, searches for health information about chronic diseases tend to be higher ${ }^{25}$.

Different scores were applied to the assessment of video quality (Fig. 3). The scores ranged from standardized scores, such as JAMA and GQS, to customized scores based on specialty guidelines and recommendations of boards, societies, and organizations. Because it was difficult to consider specific scores as they are specialty-dependent and are not unified or standardized, we focused on general scores. Even so, it has been reported that the use of both general and specific scores together to assess video quality led to consistent results ${ }^{51}$. All of the scores indicated that YouTube's health information videos were of average quality (Fig. 4). However, the use of the word "average" implies a serious problem, since it means obtaining information that is likely to have a substantial impact on health even if it is equally correct or incorrect.

We developed a quality clustering for the video based on the terms used in various papers to describe the quality categories. According to our analysis, YouTube has poor to average quality as a source of health information. In approximately $19 \%$ of papers searching for "excellent" quality videos, only $16 \%$ found videos of excellent quality. In Generally, vidoes relating to complementary and alternative medicine (CAM) were of poor quality ${ }^{52,53}$, while papers addressing specific or specialized audiences, such as trainees and residents, were of higher quality 54,55 .

The authors of several papers also assessed whether any bias existed in the making of the content (Fig. 6). Over half of them found a commercial bias in the content, mainly in information related to plastic surgery or unapproved therapies. As an example, Adeeb and colleagues (2019) study of 13 YouTube videos on "Facelift" found that 12 were created by individuals having a private practice, which is mentioned in video ${ }^{49}$. In our analysis, more than a quarter of the videos appeared to be against a particular treatment, health procedure, or health information. Only $32 \%$ of the videos were neutral, showing that YouTube videos are significantly influenced by maker bias.

Most of the studies that assessed the relationship between quality and YouTube metrics (e.g., views, likes, dislikes, shares) found no or negative correlations, indicating that YouTube metrics can be misleading indicators when it comes to healthcare video quality. Furthermore, this also demonstrates that adding 
more videos produced by professionals and health institutions will not have a significant effect without taking other measures.

As a result of our analysis, we discovered that the most frequent recommendations to improve the quality included encouraging experts and professional institutions to guide users or upload videos to YouTube. Nevertheless, YouTube is an informal educational resource which is based on different factors, such as self-motivation and general learning. In this sense, making video content will not be easy for institutions because of the limited time available to physicians and professionals. Furthermore, studies have revealed that videos uploaded by professional institutions are not necessarily more popular. According to Desai and colleague (2013), the public does not engage with videos uploaded by credible health organizations because they tend to have extensive educational content, making them overly appropriate for the public 56 .

Only $13 \%$ of papers recommended that YouTube not be used for health-related purposes. As an impractical recommendation, this recommendation ignores the present and potential benefits of YouTube in health education ${ }^{6,57,58}$ as well as the economic implications of this recommendation. A recommendation of this kind also ignores the fact that $>3.02$ billion people are expected to use social media platforms such as YouTube to seek health information by $2021^{59}$, making it nearly impossible to ignore the educational importance of these platforms.

Nearly $85 \%$ recommended improving the content - through peer review for example - but did not advise caution when using YouTube as a source of information. However, these recommendations are not specific enough, as the authors did not provide any specific steps to perform this. Moreover, this will be a complex task due to the large number of videos and the financial and technical requirements. On the other hand, about $15 \%$ of papers urged users to use caution when seeking information on YouTube. Similarly, this was unclear because no specific criteria were described for selecting and obtaining the correct information.

Collectively, we believe that the most practical step would be to create a ranking and filtering algorithm for YouTube health education videos that takes into consideration all recommendations, such as peer review and expert endorsement. The filter system should include additional parameters associated with quality. Aside from the uploading source, these criteria may also include the video duration ${ }^{60}$, presence of medical terminology (e.g., arthrocentesis versus aspiration) ${ }^{54}$, and presence of links to specialized resources in the title.. Using this filtering system, endorsed videos will be brought up and most of the recommendations made by reviewers (Fig. 8) will be taken into consideration. While a system of this type may take some time to develop, other measures can be taken, such as:

- Watching videos from more than one resource on the same topic;

- Keep in mind the bias of the author when viewing their videos;

- The presence or absence of the resources on which the videos are based; 
- To encourage healthcare institutions to periodically review YouTube for the most popular videos on health-related topics and make public announcements that specifically address misinformation.

\section{Limitations}

This study had some limitations. Firstly, we categorized the videos based on their quality. Although we believe this classification represents the data in the papers, some minor variations may occur as a result of changes made to the categories. Furthermore, we did not conduct subanalyses for each of the disease classes depicted in Fig. 2. Because of this, we were unable to determine whether YouTube content about a particular disease or category was superior to other content. Three, we limited the search to a certain time period in order to avoid bias towards COVID-19 videos, but this may result in a selection bias.

Separate studies are necessary for COVID-19 disease and vaccines against it. In addition, all the papers included in the analysis were in English. In spite of this, four papers that examined videos in languages other than English found that the results were consistent ${ }^{6,52,61,62}$. Furthermore, the protocol of this review was not pre-registered for this purpose (e.g., in PROSPERO), which may introduce potential bias in accordance with Cochrane guidelines. Finally, although we included 202 articles in our review, we may have missed some articles that we do not believe will have a significant impact on the study's findings.

\section{Conclusions And Future Work}

In this review, we presented quality analyses from 202 studies that analyzed health information from over 22,300 videos on YouTube that related to 30 medical topics. According to our study, YouTube is currently a poor or average source of health information for the general public, but videos directed at specialized audiences like residents and trainees are of higher quality. YouTube metrics are indeed tricky and can be misleading when determining the quality of videos. In the future, we recommend that a filtering system be implemented that considers expert endorsement and includes peer review.

\section{Declarations}

\section{Ethics approval and consent to participate}

Not applicable

\section{Consent for publication}

Not applicable

\section{Availability of data and material}

All data is included in the manuscript. Any request about the study design, search strategy, or any other inquiries will be addressed upon contacting the corresponding author. 
Authors declare that they have no competing interests.

\section{Funding}

There is no funding declared for this manuscript.

\section{Authors' contributions}

AS designed the study protocol with the support of WO; FA and WO separately performed the search, perfomed the analysis, and summarized the search data; WO, FA, ME, and AS interpreted the results; WO, FA, ME wrote the manuscript and ME has critically revised the manuscript. All authors have approved the submitted version of the manuscript.

\section{Acknowledgements}

The authors would like to thank Student Mona Youssef, who helped in the initial paper search collection as an independent study.

\section{Conflict of interest}

The authors declare no conflict of interest.

\section{References}

1. Statista. Hours of video uploaded to YouTube every minute as of February 2020 (https://www.statista.com/statistics/259477/hours-of-video-uploaded-to-youtube-every-minute/). (2020).

2. Syed-Abdul, S. et al. Misleading health-related information promoted through video-based social media: anorexia on YouTube. Journal of Medical Internet Research 15, e30 (2013).

3. FortuneLords. 37 Mind Blowing YouTube Facts, Figures and Statistics - 2021 (https://fortunelords.com/youtube-statistics/).

4. Statista. Percentage of adults in the United States who use selected social networks as of September 2020 (https://www.statista.com/statistics/246230/share-of-us-internet-users-who-use-selectedsocial-networks/).

5. Salama, A. et al. Consulting "Dr. YouTube": an objective evaluation of hypospadias videos on a popular video-sharing website. Journal of Pediatric Urology 16, 70. e71-70. e79 (2020).

6. Mota, P. et al. Video-based surgical learning: improving trainee education and preparation for surgery. Journal of Surgical Education 75, 828-835 (2018).

7. Infographics, G. M. I.-. YouTube User Statistics 2021 (https://www.globalmediainsight.com/blog/youtube-users-statistics/).

8. Mamlin, B. W. \& Tierney, W. M. The promise of information and communication technology in healthcare: extracting value from the chaos. The American Journal of the Medical Sciences 351, 59- 
68 (2016).

9. YouTube. Terms of Service (https://www.youtube.com/static?template=terms).

10. Nour, M. M., Nour, M. H., Tsatalou, O.-M. \& Barrera, A. Schizophrenia on YouTube. Psychiatric Services 68, 70-74 (2017).

11. Li, H. O.-Y., Bailey, A., Huynh, D. \& Chan, J. YouTube as a source of information on COVID-19: a pandemic of misinformation? BMJ Global Health 5, e002604 (2020).

12. Qi, J., Trang, T., Doong, J., Kang, S. \& Chien, A. L. Misinformation is prevalent in psoriasis-related YouTube videos. Dermatology Online Journal 22 (2016).

13. Gabarron, E., Fernandez-Luque, L., Armayones, M. \& Lau, A. Y. Identifying measures used for assessing quality of YouTube videos with patient health information: a review of current literature. Interactive Journal of Medical Research 2, e2465 (2013).

14. Madathil, K. C., Rivera-Rodriguez, A. J., Greenstein, J. S. \& Gramopadhye, A. K. Healthcare information on YouTube: a systematic review. Health Informatics Journal 21, 173-194 (2015).

15. Farag, M., Bolton, D. \& Lawrentschuk, N. Use of youtube as a resource for surgical education-clarity or confusion. European Urology Focus 6, 445-449 (2020).

16. Randolph-Krisova, A. Descriptive analysis of the most viewed YouTube videos related to the opioid epidemic. (Teachers College, Columbia University, 2018).

17. Baquero, E. P. A Descriptive Analysis of the Most Viewed YouTube Videos Related to Depression, Columbia University, (2018).

18. Aldridge, M. D. A qualitative study of the process of learning nursing skills among undergraduate nursing students. (University of Northern Colorado, 2016).

19. Foster, C. B. Mental health on Youtube: exploring the potential of interactive media to change knowledge, attitudes and behaviors about mental health, University of South Carolina, (2013).

20. Kressler, J. Women's stories of breast cancer: Sharing information through Youtube video blogs. (2014).

21. Yavuz, M. C., Buyuk, S. K. \& Genc, E. Does YouTube ${ }^{\mathrm{TM}}$ offer high quality information? Evaluation of accelerated orthodontics videos. Irish Journal of Medical Science (1971-) 189, 505-509 (2020).

22. Lee, J. S., Seo, H. S. \& Hong, T. H. YouTube as a source of patient information on gallstone disease. World Journal of Gastroenterology: WJG 20, 4066 (2014).

23. Brooks, F., Lawrence, H., Jones, A. \& McCarthy, M. YouTube ${ }^{\text {TM }}$ as a source of patient information for lumbar discectomy. The Annals of The Royal College of Surgeons of England 96, 144-146 (2014).

24. Ferhatoglu, S. Y. \& Kudsioglu, T. Evaluation of the reliability, utility, and quality of the information in cardiopulmonary resuscitation videos shared on Open access video sharing platform YouTube. Australasian Emergency Care 23, 211-216 (2020).

25. Askin, A. \& Tosun, A. YouTube as a Source of Information for Transcranial Magnetic Stimulation in Stroke: A Quality, Reliability and Accuracy Analysis. Journal of Stroke and Cerebrovascular Diseases 29, 105309 (2020). 
26. Robichaud, P. et al. Vaccine-critical videos on YouTube and their impact on medical students' attitudes about seasonal influenza immunization: a pre and post study. Vaccine 30, 3763-3770 (2012).

27. Pithadia, D. J., Reynolds, K. A., Lee, E. B. \& Wu, J. J. A cross-sectional study of YouTube videos as a source of patient information about phototherapy and excimer laser for psoriasis. Journal of Dermatological Treatment (2019).

28. Hassona, Y., Taimeh, D., Marahleh, A. \& Scully, C. YouTube as a source of information on mouth (oral) cancer. Oral Diseases 22, 202-208 (2016).

29. MacLeod, M. G. et al. YouTube as an information source for femoroacetabular impingement: a systematic review of video content. Arthroscopy: The Journal of Arthroscopic \& Related Surgery 31, 136-142 (2015).

30. Loeb, S. et al. Dissemination of misinformative and biased information about prostate cancer on YouTube. European Urology 75, 564-567 (2019).

31. Erdem, M. N. \& Karaca, S. Evaluating the accuracy and quality of the information in kyphosis videos shared on YouTube. Spine 43, E1334-E1339 (2018).

32. Kovalski, L. N. S. et al. Is the YouTube ${ }^{\text {TM }}$ an useful source of information on oral leukoplakia? Oral Diseases 25, 1897-1905 (2019).

33. Şahin, A., Şahin, M. \& Türkcü, F. M. YouTube as a source of information in retinopathy of prematurity. Irish Journal of Medical Science (1971-) 188, 613-617 (2019).

34. Castillo, J., Wassef, C., Wassef, A., Stormes, K. \& Berry, A. E. YouTube as a Source of Patient Information for Prenatal Repair of Myelomeningocele. American Journal of Perinatology (2019).

35. Camm, C. F., Russell, E., Xu, A. J. \& Rajappan, K. Does YouTube provide high-quality resources for patient education on atrial fibrillation ablation? International Journal of Cardiology 272, 189-193 (2018).

36. Joshi, S. \& Dimov, V. in World Allergy Organization Journal. A221-A221 (BioMed Central).

37. Pant, S. et al. Assessing the credibility of the "YouTube approach" to health information on acute myocardial infarction. Clinical Cardiology 35, 281-285 (2012).

38. Chen, H.-M. et al. Effectiveness of YouTube as a source of medical information on heart transplantation. Interactive Journal of Medical Research 2, e2669 (2013).

39. Brar, J., Ferdous, M., Abedin, T. \& Turin, T. C. Online information for colorectal cancer screening: a content analysis of YouTube videos. Journal of Cancer Education 36, 826-831 (2021).

40. Lenczowski, E. \& Dahiya, M. Psoriasis and the digital landscape: YouTube as an information source for patients and medical professionals. The Journal of Clinical and Aesthetic Dermatology 11, 36 (2018).

41. Gupta, N., Sandhu, G., Aggarwal, A., Singh, H. \& Leanne, F. Quality assessment of YouTube videos as a source of information on Colonoscopy. Abdomen 2 (2015). 
42. Kwok, T. M., Singla, A. A., Phang, K. \& Lau, A. Y. YouTube as a source of patient information for varicose vein treatment options. Journal of Vascular Surgery: Venous and Lymphatic Disorders $\mathbf{5}$, 238-243 (2017).

43. ElKarmi, R., Hassona, Y., Taimeh, D. \& Scully, C. YouTube as a source for parents' education on early childhood caries. International Journal of Paediatric Dentistry 27, 437-443 (2017).

44. Ovenden, C. D. \& Brooks, F. M. Anterior cervical discectomy and fusion YouTube videos as a source of patient education. Asian Spine Journal 12, 987 (2018).

45. Prabhu, V., Lovett, J. T. \& Munawar, K. Role of social and non-social online media: how to properly leverage your internet presence for professional development and research. Abdominal Radiology, 18 (2021).

46. Kunze, K. N., Krivicich, L. M., Verma, N. N. \& Chahla, J. Quality of online video resources concerning patient education for the meniscus: a YouTube-based quality-control study. Arthroscopy: The Journal of Arthroscopic \& Related Surgery 36, 233-238 (2020).

47. Wong, K., Doong, J., Trang, T., Joo, S. \& Chien, A. L. YouTube videos on botulinum toxin A for wrinkles: a useful resource for patient education. Dermatologic Surgery 43, 1466-1473 (2017).

48. Felgoise, S., Monk, M. \& Gentis, K. Information Shared on YouTube by Individuals Affected by Long QT Syndrome: A Qualitative Study. (2016).

49. Derakhshan, A., Lee, L., Bhama, P., Barbarite, E. \& Shaye, D. Assessing the educational quality of 'YouTube'videos for facelifts. American Journal of Otolaryngology 40, 156-159 (2019).

50. Keelan, J., Pavri-Garcia, V., Tomlinson, G. \& Wilson, K. YouTube as a source of information on immunization: a content analysis. JAMA 298, 2482-2484 (2007).

51. Kanlioz, M. \& Ekici, U. Reliability and educational features of YouTube videos about hernia operations performed using laparoscopic TEP method. Surgical Laparoscopy, Endoscopy \& Percutaneous Techniques 30, 74-78 (2020).

52. Lee, T. H. et al. Medical professionals' review of YouTube videos pertaining to exercises for the constipation relief. The Korean Journal of Gastroenterology 72, 295-303 (2018).

53. Gonzalez-Estrada, A. et al. in Allergy \& Asthma Proceedings.

54. Fischer, J., Geurts, J., Valderrabano, V. \& Hügle, T. Educational quality of YouTube videos on knee arthrocentesis. JCR: Journal of Clinical Rheumatology 19, 373-376 (2013).

55. Abdulghani, H. M. et al. A critical review of obstetric and gynecological physical examination videos available on YouTube: Content analysis and user engagement evaluation. Medicine 98 (2019).

56. Desai, T. et al. Is content really king? An objective analysis of the public's response to medical videos on YouTube. PLOS One 8, e82469 (2013).

57. Knösel, M., Engelke, W., Helms, H. J. \& Bleckmann, A. An appraisal of the current and potential value of web 2.0 contributions to continuing education in oral implantology. European Journal of Dental Education 16, 131-137 (2012). 
58. Tulgar, S. Quality of Erector Spinae Plane Block Educational Videos on a Popular Video-sharing Platform. (2019).

59. Huo, J. et al. Use of social media in health communication: findings from the Health Information National Trends Survey 2013, 2014, and 2017. Cancer Control 26, 1073274819841442 (2019).

60. Frongia, G. et al. YouTube as a potential training resource for laparoscopic fundoplication. Journal of Surgical Education 73, 1066-1071 (2016).

61. Ruppert, L. et al. YouTube as a source of health information: Analysis of sun protection and skin cancer prevention related issues. Dermatology Online Journal 23 (2017).

62. Sabra, M. A., Kamel, A. A. \& Malak, M. Z. Alzheimer disease health-related information on YouTube: A video reviewing study. Journal of Nursing Education and Practice 6 (2016).

\section{Figures}




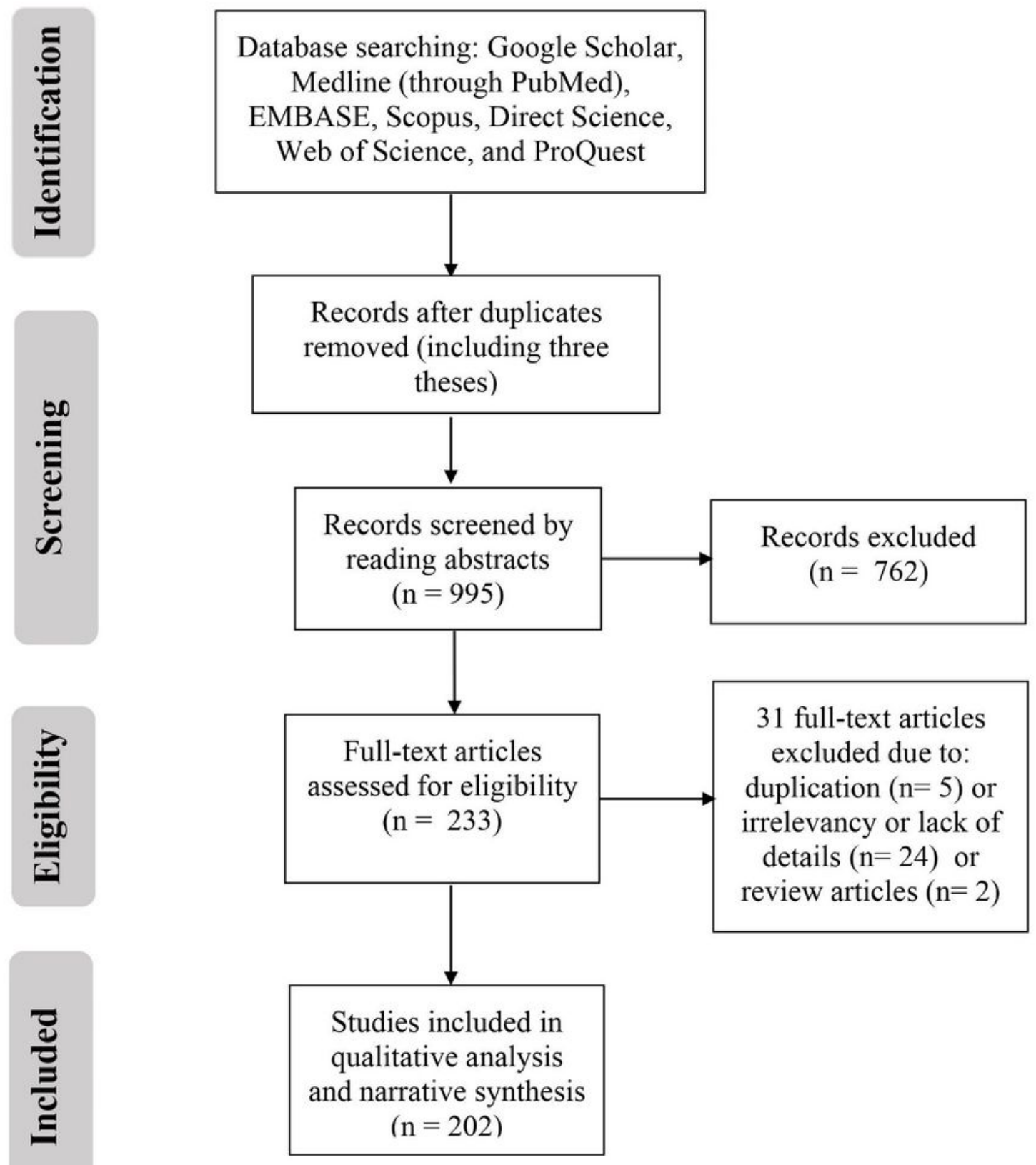

Figure 1

PRISMA Flow chart of literature screening and selection for reviewed articles 


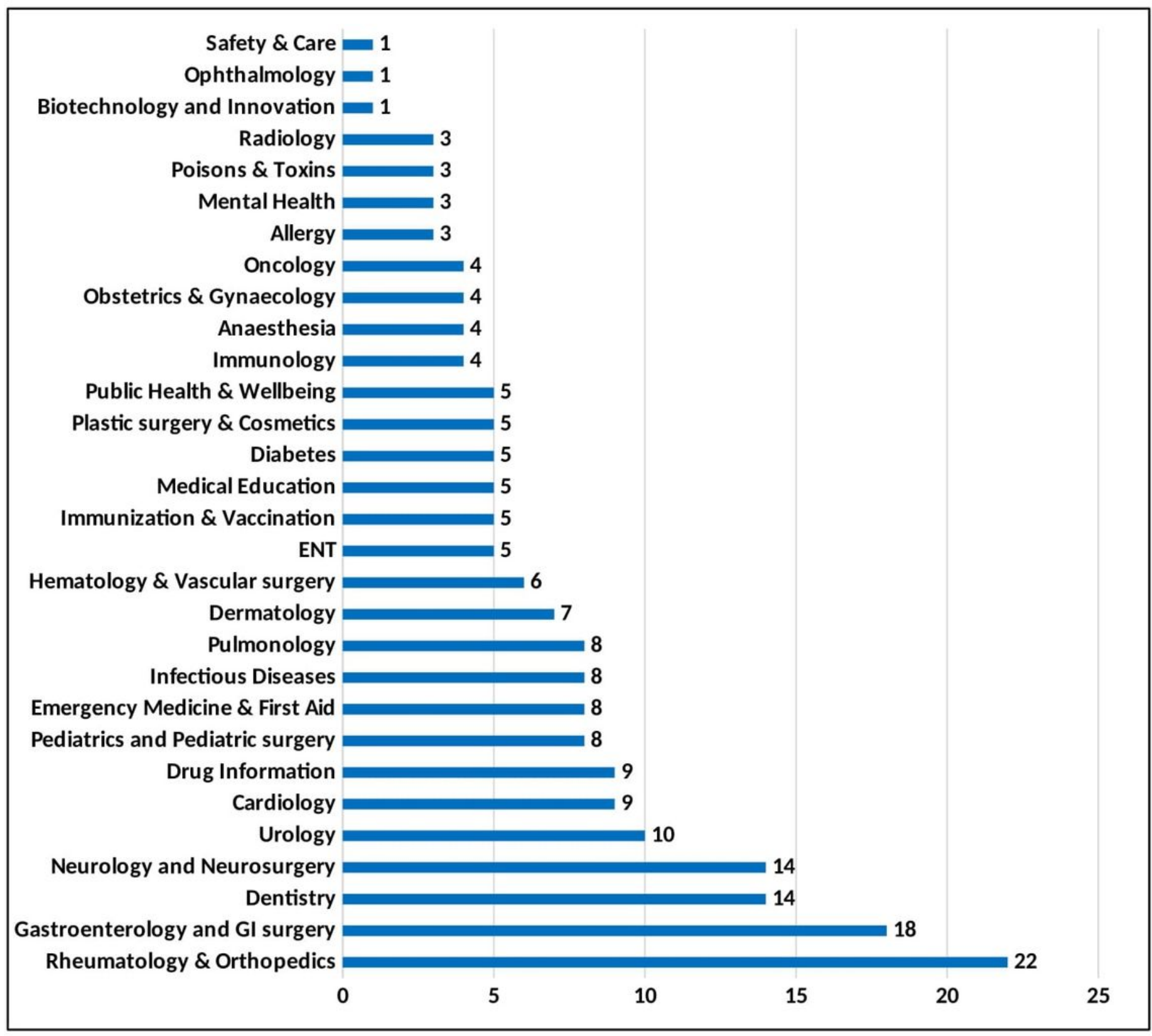

Figure 2

Fields addressed by reviewed studies and number of papers in the respective field 


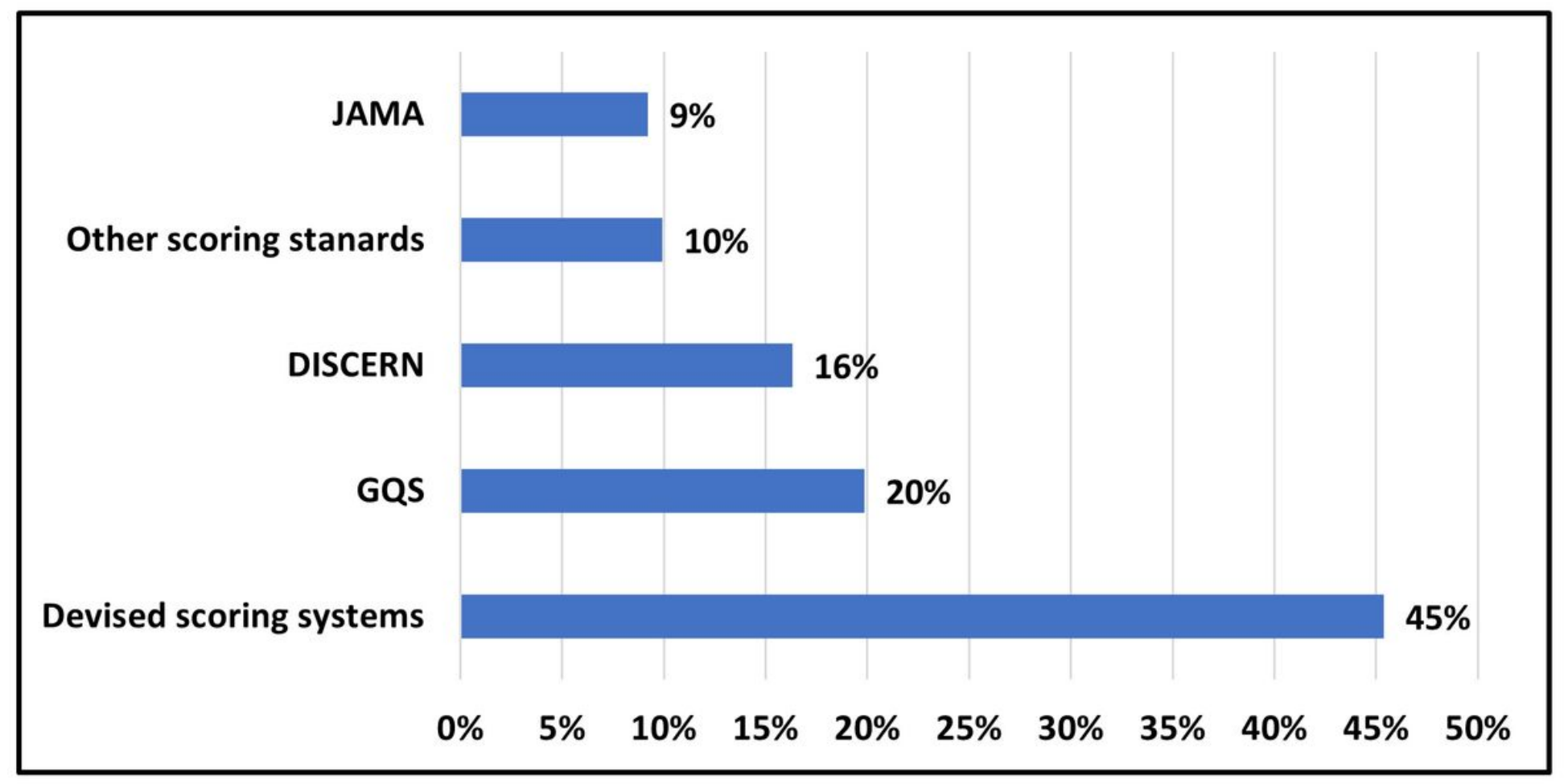

Figure 3

Common scoring systems for assessing the content's quality

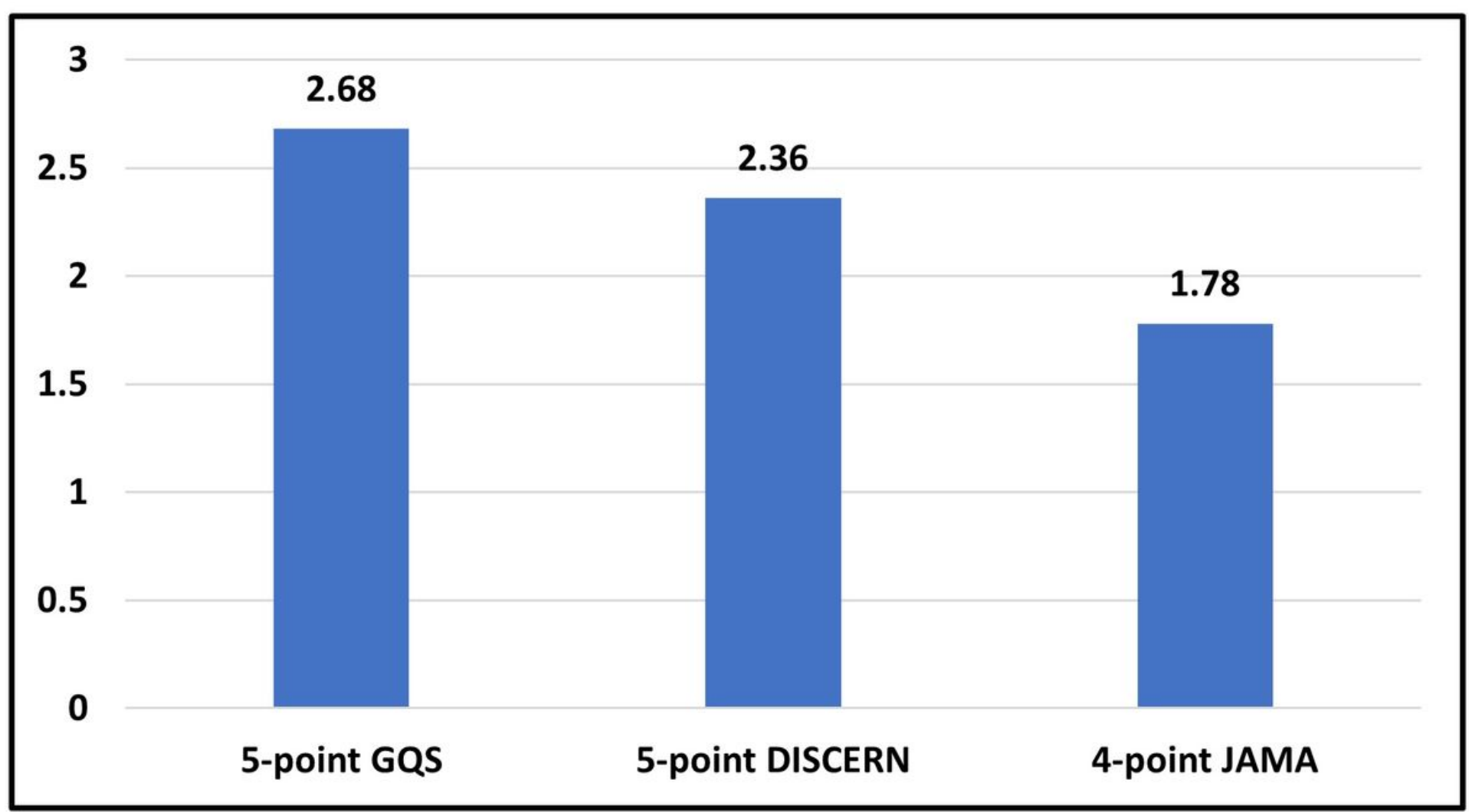

Figure 4 
The average quality measures from three general standard scores: GQS, JAMA, and DISCERN

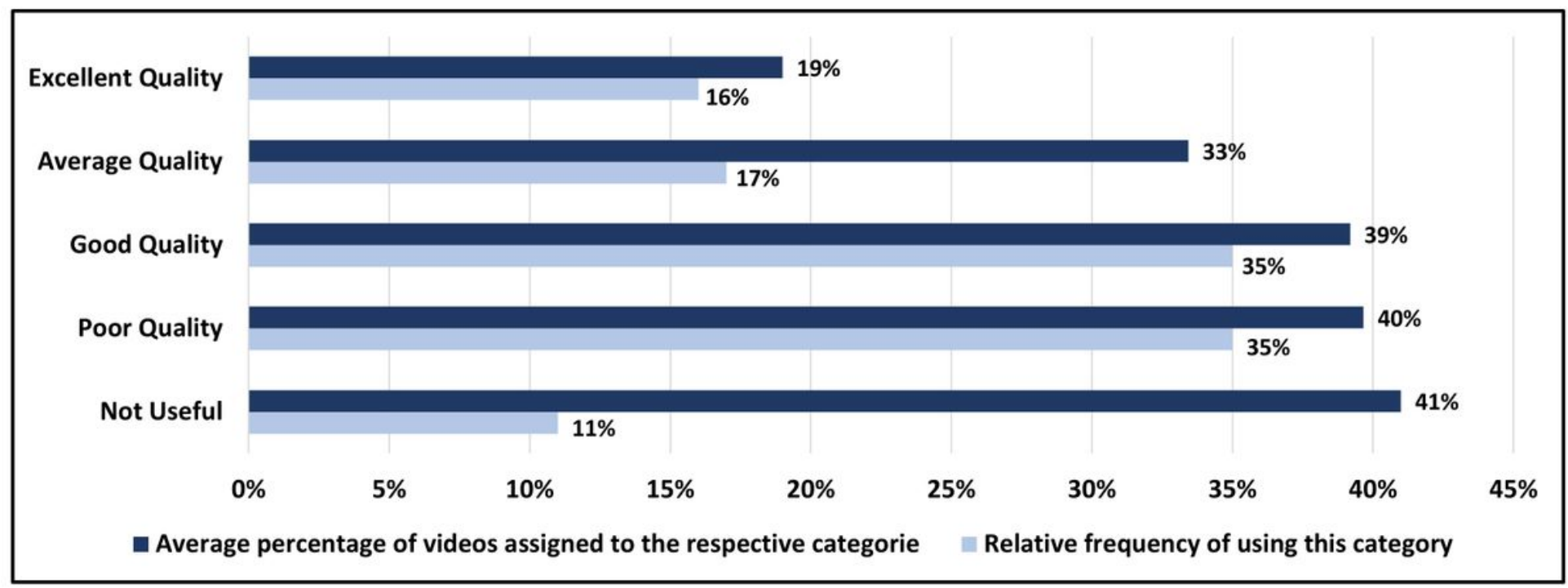

Figure 5

The most common quality categories for the content as used in the reviewed papers

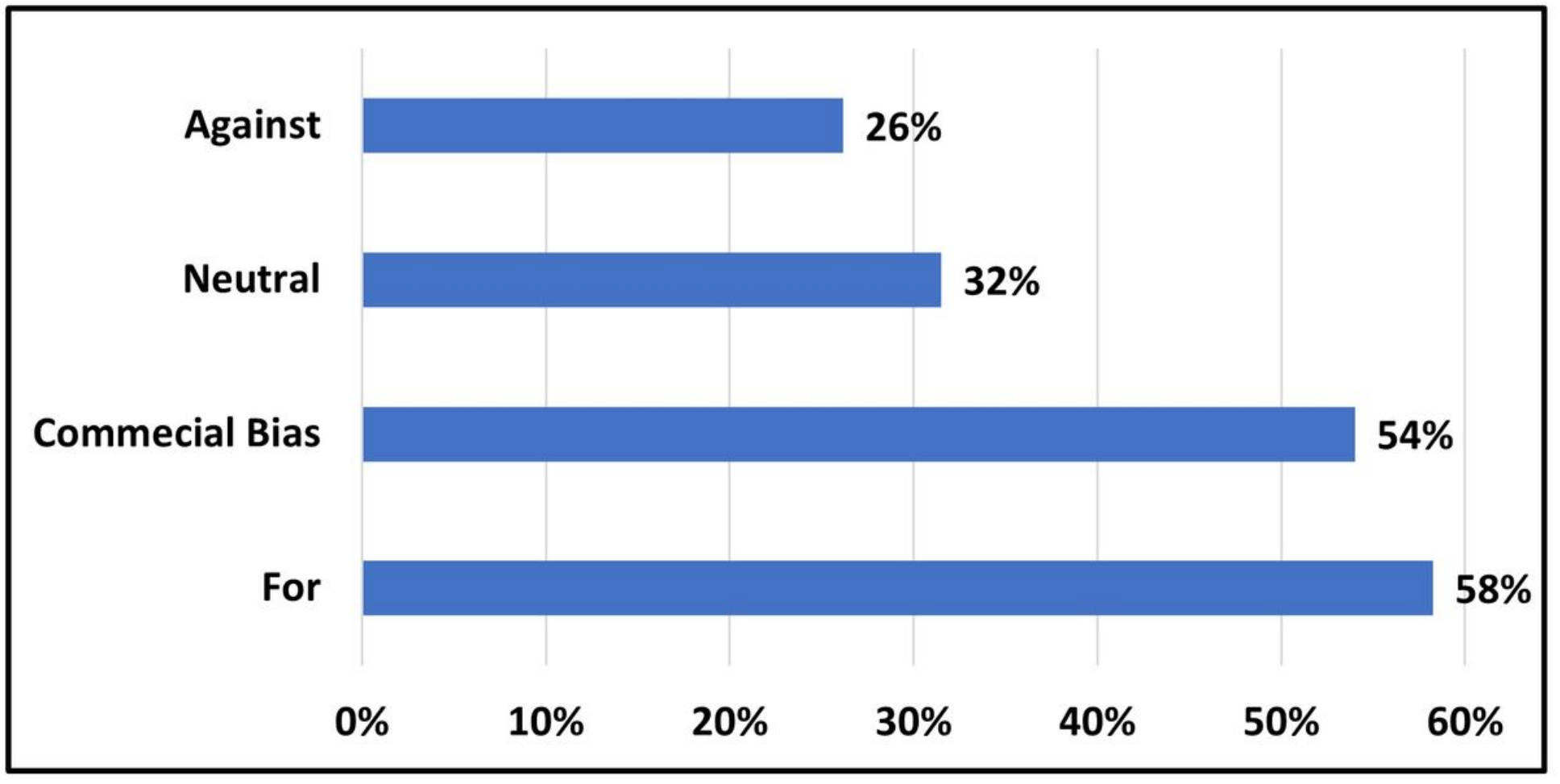

Figure 6

The classification of the reviewed papers according to bias classes 
20

15
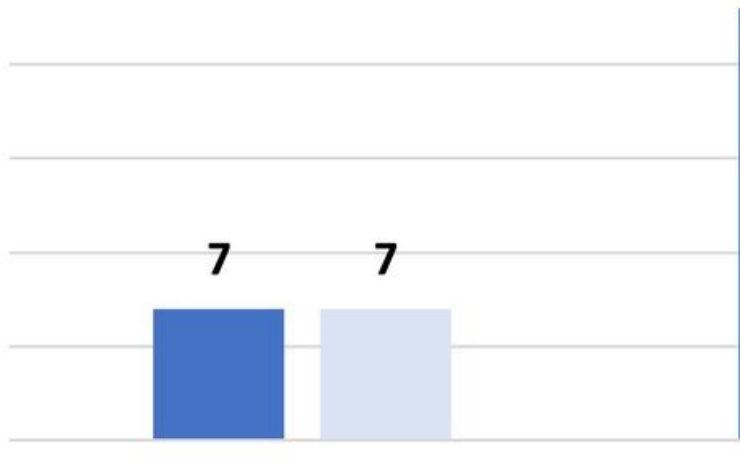

Positive correlation

No correlation

13

10
5
0

Video quality vs. the number of views Video quality vs. the number of likes

Figure 7

The correlation between video quality and popularity as described in the reviewed papers

YouTube is a good source for health-related information
YouTube's ranking and flitration system should be improved
Content on YouTube should undergo a peer review process

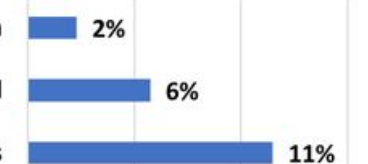

YouTube should not be used for seeking health-related information

Users should exercise caution when seeking information on YouTube

Experts should guide users by recommending selected content

Professional institutions should upload more qualified content
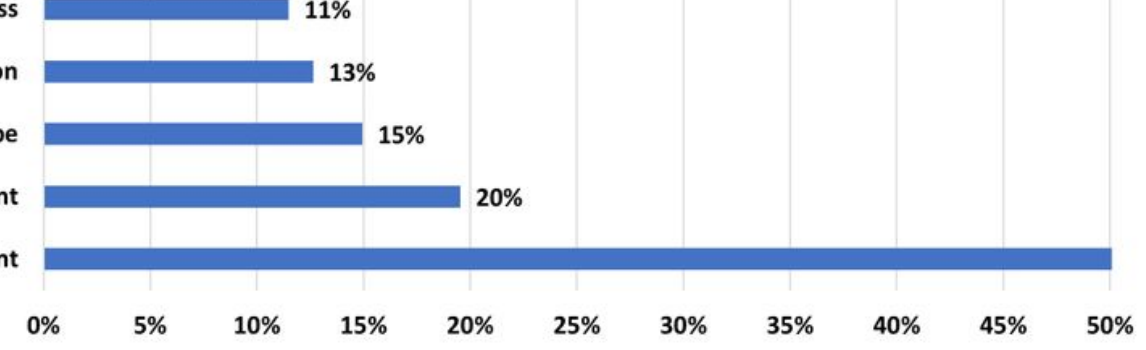

\section{Figure 8}

Recommendations derived from the reviewed papers and their frequency

\section{Supplementary Files}

This is a list of supplementary files associated with this preprint. Click to download.

- SupplementaryTable1WaelOsmanetal..pdf 